\title{
Pressure Dependence of Frequencies and Frequencies Distribution Function for Aluminum: Theoretical approach
}

\author{
Seham J. Abdallah
}

Adnan M. Al-sheikh

Department of physics

College of science

University of Mosul

Received

$13 / 12$ / 2007
Accepted

07 / 04 / 2008

\section{الملخص}

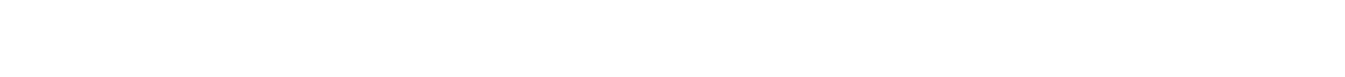

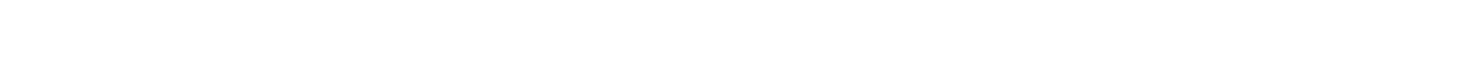
الفونوني للألمنيوم.

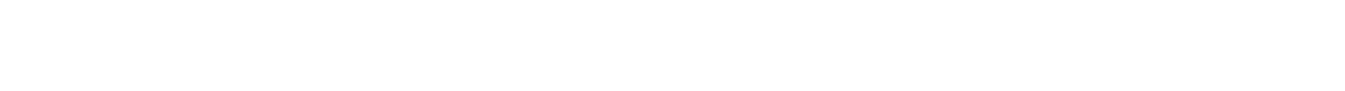

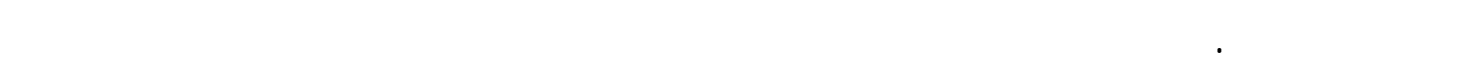
كرونشين ومعادلات الحالة لمرنكهان، برخ -مرنكهان ومعادلة فينت.

\begin{abstract}
A theoretical study was made of the pressure dependence of lattice frequencies and distribution function for aluminum by analyzing phonon frequency spectrum, using Grüneisen approximation and many equations of state (EOS).

Each change in the specific volume of a crystal excites a change in the frequencies of lattice vibration in a very complex manner. This work shows how this complexity can be overcome at least approximately depending on Grüneisen approximation and Murnaghan , Birch- Murnaghan and Vinet equations of state.
\end{abstract}




\section{Introduction:}

Materials at high pressures occur at centers of planets and in stars and in both natural and man made explosions. High pressures may also be applied to small laboratory samples in a controlled manner using devices such as the diamond anvil cell (DAC). The static pressure applied in $\mathrm{DAC}$ is a continuously variable parameter which can be used for systematic studies of the properties of solids as a function of the interatomic distance [1].

One of the interesting phenomena that may occur under applied pressure is a sudden change in the arrangement of the atoms. The Gibbs free energies of the different possible arrangements of atoms vary under compression, and at some stage it becomes favorable for the material to change the type of atomic arrangement [2]. A phase transition is said to have occurred if the change is discontinuous or continuous but with a change in crystal symmetry.

The pressures achieved in a DAC can lead to a reduction in the volume, causing enormous changes to the inter-atomic bonding. The properties of the high-pressure phases may be very different from those under normal conditions.

The equation of state (EOS) of a system describes the relation among thermodynamic variables such as pressure, temperature and volume. It provides numerous pieces of information relating to the nonlinear compression of a material at high pressure, and has been widely applied in engineering and other scientific research. Recently, rapid advances in computational capabilities and accurate high pressure experimental techniques have given a strong impetus to theoretical work [3]. In present work an evaluation of the effect of pressure on lattice varibration and mode density for (Al) has been achieved. A suitable approximation of the change in frequencies due to change in specific volume of a crystal, the Grüneisen approximation, using Murnagha EOS, Birch-Murnghan EOS and Vinet EOS.

\section{Theoretical Details :-}

\subsection{Equation of state :-}

\subsubsection{Murnaghan Equation of state :-}

In the Murnaghan approximation, an isothermal relation between pressure $(\mathrm{P})$ and volume $(\mathrm{V})$ for solids is derived from the assumption that isothermal bulk modulus (B) is a linear function of P. [4]. Make a Taylor expansion of the adiabatic bulk modulus [5] 


$$
\begin{aligned}
B_{s} & =B_{o s}+B^{\prime} P \ldots \ldots \ldots \ldots \ldots . . . \\
& =B_{o s}\left(1+\frac{B^{\prime}}{B_{o s}} p\right) \\
& =-V\left(\frac{\partial P}{\partial V}\right)_{s}
\end{aligned}
$$

$\mathrm{B}_{\mathrm{s}}$ - Adiabatic bulk modulus at pressure

$\mathrm{B}_{\mathrm{os}}$ - adiabatic bulk modulus at atmosphere pressure

$\mathbf{B}_{s}^{\prime}$ - Pressure bulk modulus derivative(adiabatic), dimensionless

$$
\begin{aligned}
& \int_{V o}^{V p}-\frac{d V}{V}=\frac{1}{B_{o s}} \int_{0}^{P} \frac{d P}{1+\frac{B^{\prime} P}{B_{o s}}} \\
& \operatorname{Ln} \frac{V_{o}}{V_{p}}=\frac{1}{B_{o s}}\left[\frac{B_{o s}}{B_{s}^{\prime}} \operatorname{Ln}\left(1+\frac{B_{s}^{\prime} P}{B_{o s}}\right)\right]_{0}^{P} \\
& \operatorname{Ln} \frac{V_{o}}{V_{p}}=\operatorname{Ln}\left(1+\frac{B_{s}^{\prime} P}{B_{o}}\right)^{\frac{1}{B_{s}^{\prime}}} \\
& \frac{V_{p}}{V_{o}}=\left(1+\frac{B_{s}^{\prime} P}{B_{o s}}\right)^{\frac{-1}{B_{s}^{\prime}}}
\end{aligned}
$$

\subsubsection{Birch-Murnaghan EOS [6] :-}

This EOS based upon the assumption that the strain energy of a solid undergoing compression can be expressed as a Taylor series in the finite strain.

$$
P(V)=\frac{3}{2} B_{o T}\left[\left(\frac{V_{o}}{V_{p}}\right)^{7 / 3}-\left(\frac{V_{o}}{V_{p}}\right)^{5 / 3}\right]\left[1-\frac{3}{4}\left(4-B_{T}^{\prime}\right)\left\{\left(\frac{V_{o}}{V_{p}}\right)^{2 / 3}-1\right\}\right]
$$

$\mathrm{B}_{\mathrm{OT}^{-}}$thermal bulk modulus at atmosphere pressure $B_{T}^{\prime}$ - Pressure bulk modulus derivative, dimensionless 


\subsubsection{Vinet $\operatorname{EOS}([7] \&[8])$ :-}

This EOS derived from a general inter-atomic potential [9]. For simple solids under very high compressions the resulting Vinet EOS provides a more accurate representation of the volume variation with pressure.

$P=3 B_{o T}\left(\frac{V_{p}}{V_{o}}\right)^{-2 / 3}\left(1-\left(\frac{V_{p}}{V_{o}}\right)^{1 / 3}\right] \exp \left[\frac{3}{2}\left(B_{T}^{\prime}-1\right)\left\{1-\left(\frac{V_{p}}{V_{o}}\right)^{1 / 3}\right\}\right]$

\section{2- Grüneisen parameter :}

The Grüneisen parameter is of great importance for thermal equation of state of materials at high pressures, as it treats thermal effects at high pressures.

In the Mie - Grüneisen theory of thermal expansion of solids, the Grüneisen parameter $\gamma$ is defined by [10].

$$
\gamma=\frac{\alpha_{V} \cdot B_{T}}{C_{V} \cdot \rho}=\frac{\alpha \cdot B_{s}}{C_{P} \cdot \rho}
$$

Where

$\alpha_{\mathrm{V}}$-volume coefficient of thermal expansion

$\mathrm{B}_{\mathrm{T}}$ - isothermal bulk modulus

$\mathrm{C}_{\mathrm{p}}, \mathrm{C}_{\mathrm{v}}$ - are the specific heat at constant pressure and constant volume.

$\rho$ - density

The connection between the frequencies $v$ and $v_{p}$ of corresponding vibrations at specific volumes $\mathrm{V} \& \mathrm{~V}_{\mathrm{p}}$, using Grüneisen approximation, given by the following expression [11].

$$
\frac{v_{p}}{v}=\left(\frac{V_{p}}{V}\right)^{-\gamma}
$$

By considering the physical significance of the distribution function of the frequencies, the relation between the function $g(v, v)$ for a volume $\mathrm{V}$ and the analogical function $\mathrm{g}\left(\mathrm{v}, \mathrm{V}_{\mathrm{p}}\right)$ for a volume $\mathrm{V}_{\mathrm{p}}$ is .

$$
g\left(v, V_{p}\right)=\left(\frac{V_{p}}{V}\right)^{\gamma} g\left[v\left(\frac{V_{p}}{V}\right)^{\gamma}, V\right]
$$




\section{Computation and Results:}

The frequency distribution function of aluminum (Fig. 1) had been calculated by [12] base on the Z- neighbor model in which the interatomic forces are restricted to interactions with the first and second neighbors.

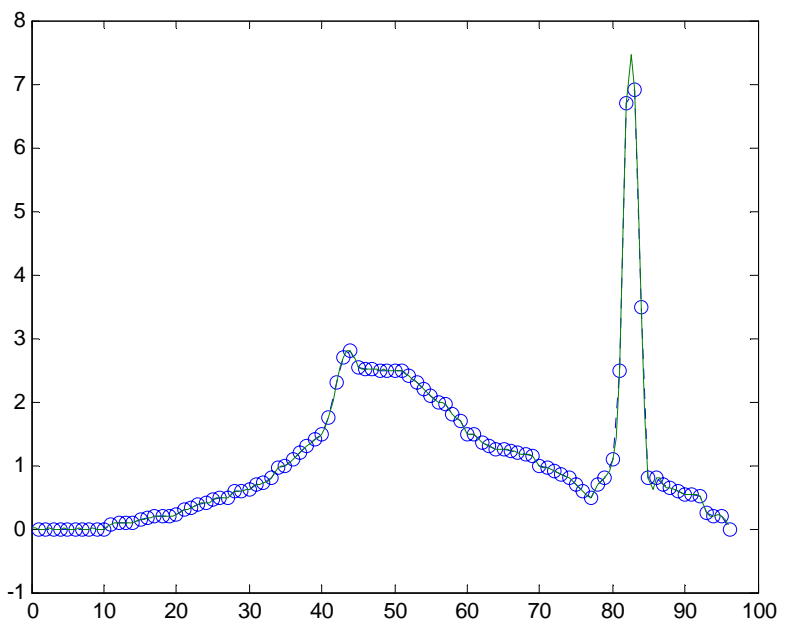

Fig.1: phonon frequency spectrum for Aluminum[12]

The above pfs has been calibrated using Matlab 7.5 (spline) where the corresponding $\mathrm{g}(\mathrm{v})$ value for each $\mathrm{v}$ is obtained. (Gandoin \& Foulks, 2002)[13] gave the value of bulk modules for $\mathrm{Al}(\mathrm{B}=813 \mathrm{kbar})$, and its pressure derivative $\left(\mathrm{B}^{\backslash}=4.27\right)$. Hence Murnaghan EOS of state for $\mathrm{Al}$ can be expressed in the form.

$$
\frac{V_{P}}{V}=\left(1+\frac{P}{190.4}\right)^{-0.234}
$$

Combining equations 8 and 6 , one obtain

$$
v_{P}=v\left(1+\frac{P}{190.4}\right)^{+0.234 \gamma}
$$

$$
\text { Where } \gamma=2.14 \text { for Al [14]. }
$$

Equation (9) represent the effect of pressure on lattice vibration. (Fig.2) illustrates frequencies shift to higher frequencies for certain pressure in addition to represent $\mathrm{Al}$ phonon frequency spectra at different pressures. 


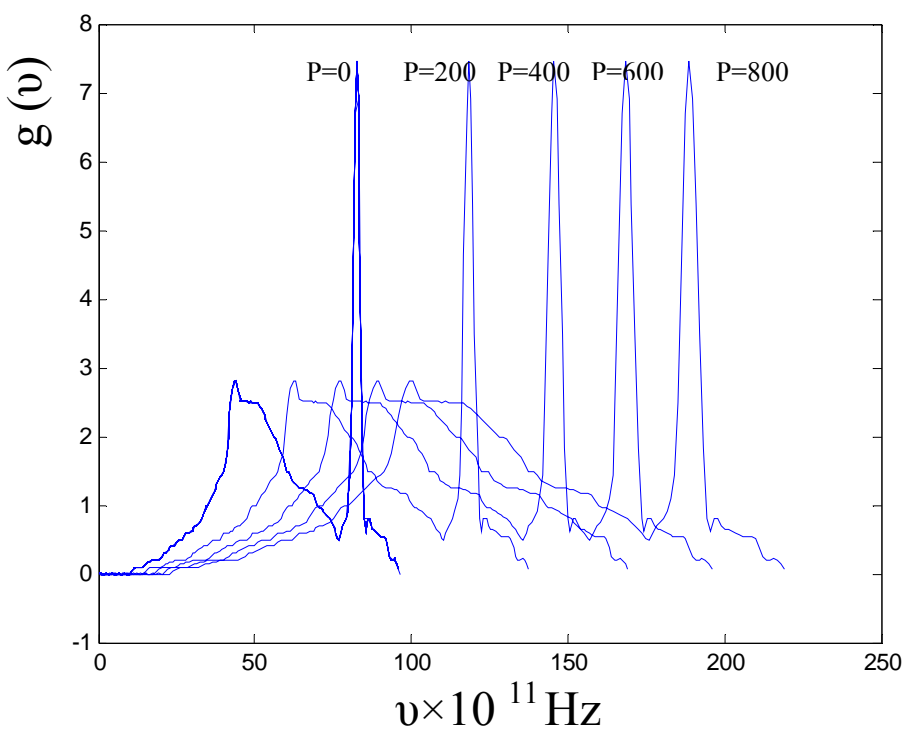

Fig.2: Variation of frequencies, only, with pressure (kbar) for aluminum pfs using Murnaghan EOS.

The dependence of the distribution function on specific volume for $\mathrm{Al}$ evaluated by equation (10) which obtained from equations( 7 and 8).

$g\left(v, V_{P}\right)=\left(1+\frac{P}{190.4}\right)^{-0.234 \gamma} g\left[v\left(\frac{V_{P}}{V}\right)^{\gamma}, V\right]$

(Fig.3) show the variation of $g(v)$ only with pressure, while (Fig.4) shows the effect of pressure on both $(v)$ and $g(v)$ simultaneously for different values of pressures.

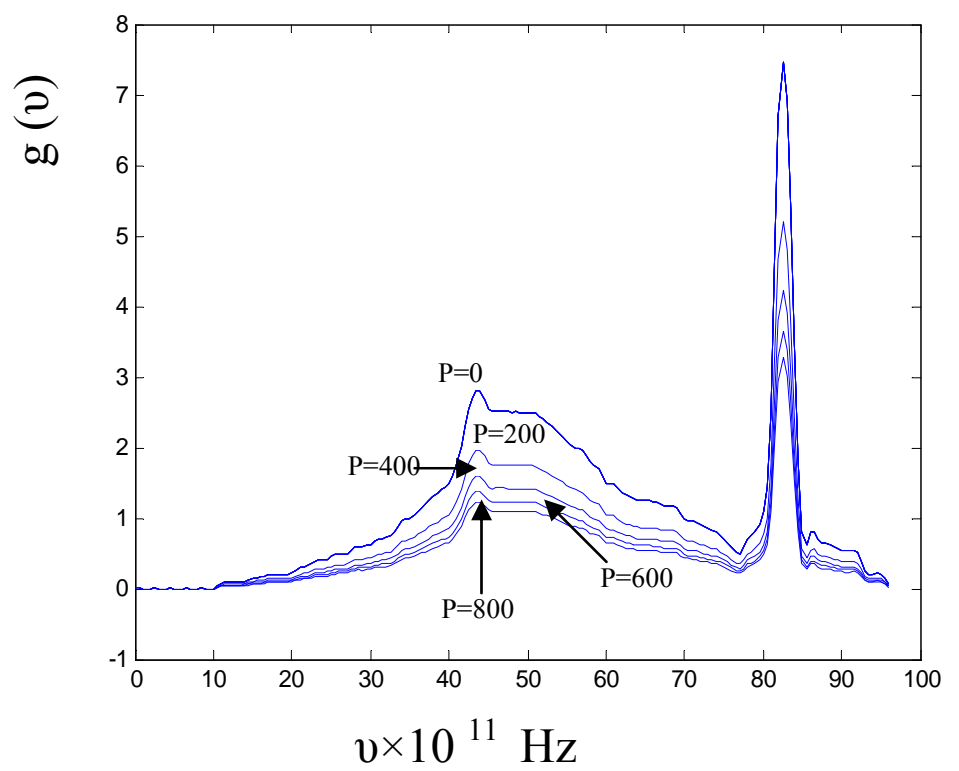

Fig.3: Variation of mode density, only, with pressure (kbar) for aluminum, using Murnaghan EOS. 


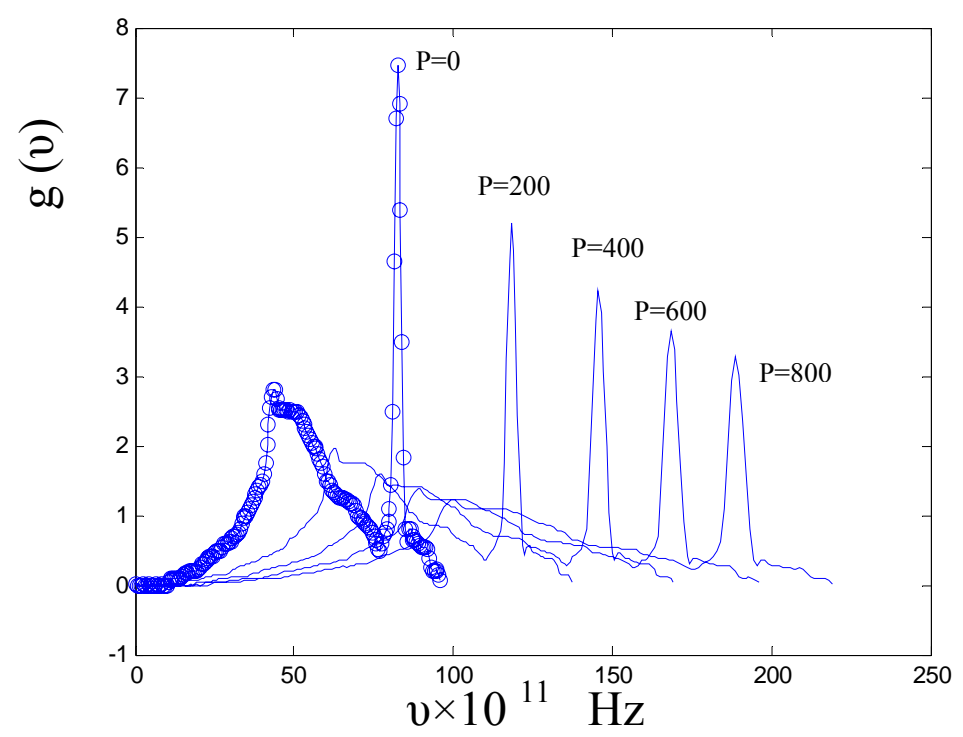

Fig.4: Variation of both $v$ and $g(v)$, simultaneously with pressure (kbar) for aluminum, using Murnaghan EOS.

Using equations ( 3 and 4) with $\mathrm{B}_{\mathrm{oT}}=727(\mathrm{kbar})$ and $\mathrm{B}_{\mathrm{T}}=4.14$ [2]. Figs(5 and 6) represent the variation of pfs for $\mathrm{Al}$ with pressure using Birch EOS and Vinet EOS respectively.

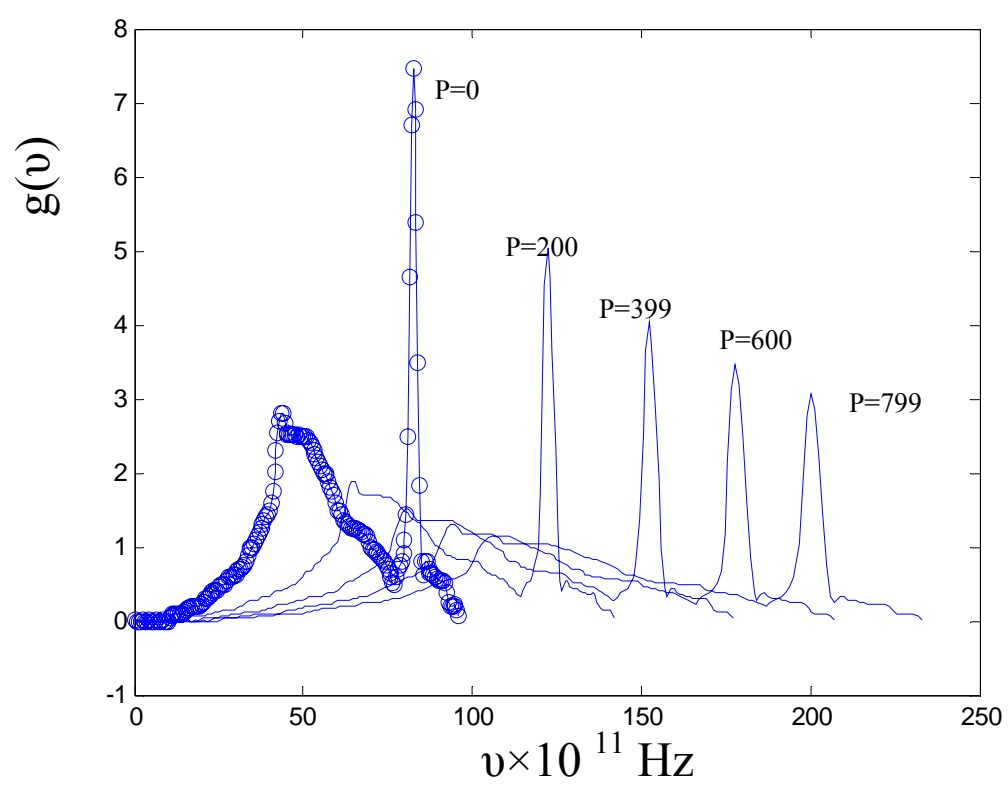

Fig.5: Variation of both $v$ and $g(v) ;$ simultaneously with pressure (kbar) for aluminum, using Birch-Murnaghan EOS 


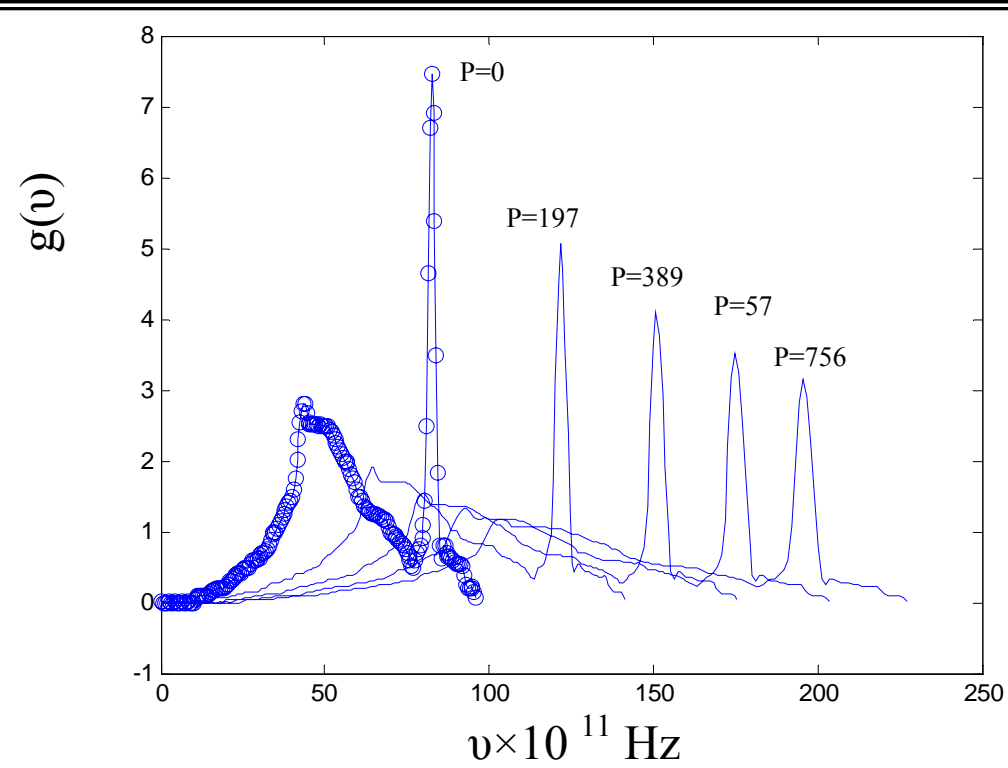

Fig.6: Variation of both $v$ and $g(v)$,simultaneously with pressure (kbar) for aluminum, using Vinet EOS

Table-1 summarize comparison for variation, with pressure, of aluminum lattice frequencies and its mode density, using Murnghan, BirchMurnghan and Vinet equations of state.

\begin{tabular}{|c|c|c|c|c|c|c|c|c|}
\hline \multicolumn{4}{|c|}{ Murnghan EOS } & \multicolumn{3}{c|}{ Birch-Murnghan EOS } & \multicolumn{3}{c|}{ Vinet EOS } \\
\hline $\mathrm{P}(\mathrm{kbar})$ & $v_{\max }$ & $\mathrm{g}_{\max }(\mathrm{v})$ & $\mathrm{P}(\mathrm{kbar})$ & $v_{\max }$ & $\mathrm{g}_{\max }(\mathrm{v})$ & $\mathrm{P}(\mathrm{kbar})$ & $v_{\max }$ & $\mathrm{g}_{\max }(\mathrm{v})$ \\
\hline 0 & 96 & 7.462 & 0 & 96 & 7.462 & 0 & 96 & 7.462 \\
\hline 200 & 137.6 & 5.206 & 200 & 142.2 & 5.037 & 197 & 141.6 & 5.058 \\
\hline 400 & 169.3 & 4.232 & 399 & 177.2 & 4.043 & 389 & 175.6 & 4.08 \\
\hline 600 & 195.9 & 3.656 & 600 & 206.9 & 3.462 & 576 & 203.6 & 3.519 \\
\hline 800 & 219.4 & 3.265 & 799 & 232.8 & 3.077 & 756 & 227.4 & 3.149 \\
\hline
\end{tabular}

Table-1: Variation of Al lattice vibrations and mode density with pressure using different EOS.

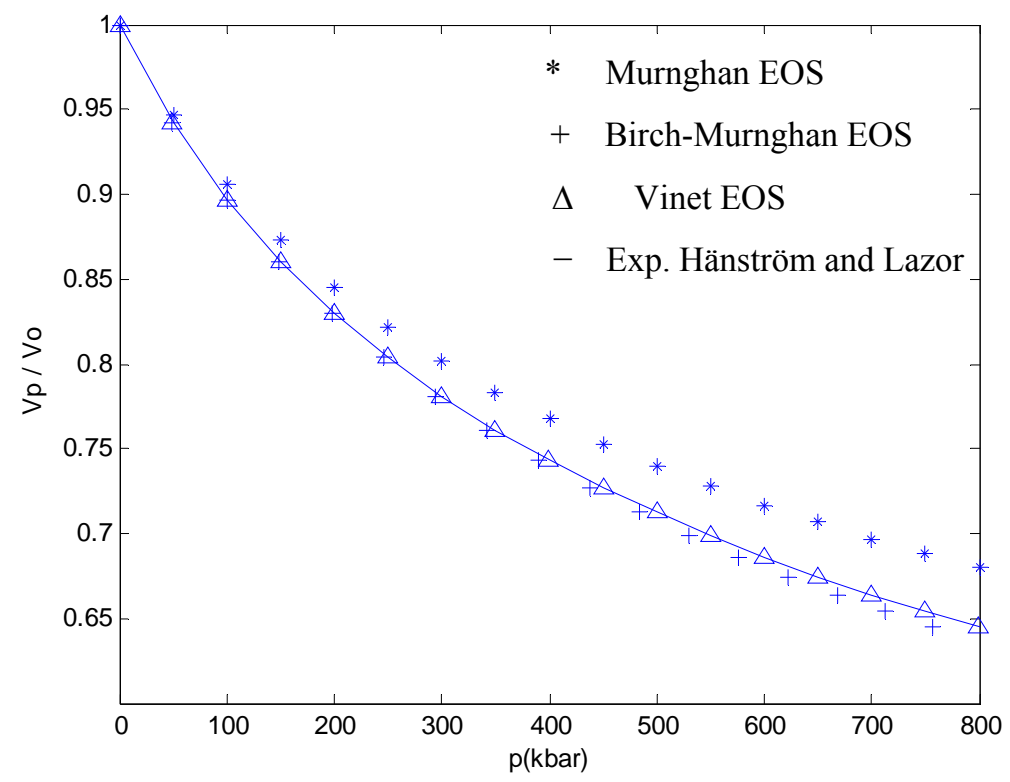

Fig.7: Variation of aluminum $\left(\mathrm{V}_{\mathrm{p}} / \mathrm{V}_{\mathrm{o}}\right)$ with pressure using different EOS as compared with experimental data. 


\section{Discussion :}

High pressure induce change in lattice constant and consequently in specific volume of a crystal [15] .Each change in the specific volume of a crystal excites a change in the equilibrium position of the atoms and in turn a change in the frequency spectrum .Even if we confine ourselves to isotropic changes in volume the frequencies of vibrations will change as a function of change in specific volume in a very complex manner. If, however, we consider all the difficulties in calculating the frequency spectrum of a crystal, it is unthinkable to perform further calculations for the different specific volumes. This work shows how these difficulties can be overcome at least approximately depending on Grüneisen approximation and Murnaghan EOS, Birch-Murnghan and Vinet EOS, especially that this approximation gives a reasonable interpretation [16] for the high pressure data of silver [17].

Although all the three EOS shows different values shift to higher frequencies and decreasing in $\mathrm{g}(\mathrm{v})$ at high pressure we suggest that BirchMurnghan EOS represent the best results for variation of Al pfs with pressure as this equation gives the best fit for variation of specific volume with pressure as shown in Fig. (7). 


\section{References:}

1. Mujica A, Rubio A., Munoz A and Needs R. J., Rev. Mod. phys., 75:863 -912 (2003).

2. Hänström A. and Lazor P., J .alloys and comp., 305 :209 -215(2000).

3. Jiuxum S, J. phys., condens. Matter 17: L 103 -L 111 (2005).

4. Guillermet F., Int. J. Thermody., 16, No.4 :1009-1026 (1995).

5. Weisstein Eric., Word of phys., science world wolfram.com (1989) Birch F., phys Rev., 71:809-824 (1947).

6. Vinet P., Ferrante J., Smith JR., Rose JH., J phys C., solid state 19: L 467 -L 473 (1986).

7. Vinet P., Ferrante J., Smith JR., Rose JH., Geophys Res 92: 9319 9325 (1987).

8. Angel R. J, EOS fit 6.0 users guide, www.geol.Vt.edu/profs/ria/ . Crystallography Laboratory, Dept. Geological sciences, Virginia Tech, Black sburg, VA 24060, USA (June 2001).

9. Grüneisen (1912) cited in Ref.14.

10. Dlouha J., J. phys., B14:571 -579 (1964).

11. Lee T. chen and Hwangi J-Lin., Chinese J. phys., 7, No.1:33-39 (1968).

12. Gaudion R. and Foulkes W. M. C., phys. Rev. B, 052104:1 -4(2002).

13. Boehler R. and Ramakrishnan J., J. Geophys. Res., 85, No.B12:6996-7002 (1980).

14. Radi I. O., Abdulsttar M. A. and Abdul-Lettif A.M., phys. state. sol. (b) 244, No.4:1304-1317 (2007).

15. Ahmed J. F. AL-Deen, ph. D. theseis, college of Education, University of Mosul (2007). (In Arabic)

16. Xie J. de Gironcoli S., Baroni S., and Scheffler M., phys. Rev. B,59, No.2 (1999).

17. Xie J. de Gironcoli S., Baroni S., and Scheffler M., phys. Rev. B,59, No.2 (1999). 